\title{
Kritik der postkritischen Pädagogik
}

\author{
Hauke Brunkhorst
}

Es gab in den 1960er und 1970er Jahren eine explizit kritische Erziehungswissenschaft, die sich auch als emanzipatorische Pädagogik verstand und Motive der Frankfurter Schule (»kritische Theorie«) pädagogisch ausbuchstabiert hat. Klaus Mollenhauers »Polemische Skizzen«, die er 1968 unter dem Titel »Erziehung und Emanzipation« publizierte, sind immer noch einschlägig, aber auch die Arbeiten von Ilse Dahmer, Heinz-Joachim Heydorn, Hans-Jochen Gamm und Gernot Koneffke begraben die konservativ geprägte und faschistisch kontaminierte Epoche der geisteswissenschaftlichen Pädagogik und begründen eine neue gesellschaftskritische Bildungstheorie (Mollenhauer 1968; Dahmer/Klafki 1968; Heydom 1995). Die von Eduard Bernstein bis Anton Semjonowitsch Makarenko reichenden, in der Weimarer Republik entstandenen linken, sozialistischen, psychoanalytischen und marxistischen Schulen der Sozialisationstheorie, der Erziehungswissenschaft und -praxis werden wiederentdeckt, weiterentwickelt und neu vernetzt, nachdem sie 1933 unterdrückt und nach 1945 im Zeichen des Antikommunismus verdrängt worden sind. Wilhelm Reich dringt in die Oberstufen der Gymnasien und den Campus der Universitäten ein und revolutioniert Sexualpolitik und Sexualpädagogik. Das Private »machtgeschützte Innerlichkeit « (Mann 1918/2001) - wird im Zeichen der Frauenbewegung politisch und der Weiberrat des SDS befreit die "sozialistischen Eminenzen von ihren bürgerlichen Schwänzen« (Miemeister/Staadt 1980: 223f.). Die zeitgenössische antiautoritäre 
Pädagogik von Alexander Sutherland Neill (Summerhill) bis zur Kinderladenbewegung kommt der kritischen Erziehungswissenschaft ebenso entgegen wie der breite Diskurs um die Bildungs- und Hochschulreform, die insbesondere durch die SDS-Denkschrift von 1961: Hochschule in der Demokratie vorangetrieben und zur wirkmächtigen Alternative der technokratischen Hochschulreform wurde. Nicht zuletzt hat die von Basil Bernsteins linguistischen Forschungen ausgehende Idee kompensatorischer Erziehung der wachsenden Kritik an der Reproduktion der Klassengesellschaft durch das Schul- und Erziehungssystem den Rücken gestärkt und in den davon unabhängigen Studien Pierre Bourdieus zum symbolischen Kapital eine späte, bis heute reichende Fortsetzung erfahren. »Mehr Demokratie wagen« (Willi Brandt) wurde 1969 zum politischen Programm, wurde mit verschiedenen Varianten des Sozialismus verbunden, wurde zur Parole umfassender Bildungsreformen (Gesamtschule, Gesamthochschule, Drittelparität, Projektunterricht, aber auch in der Sozialpädagogik: "Staffelberg«, Heimerziehung usw.). Und auch aus den Ländern des globalen Südens kamen die Befreiungstheologie und die Pädagogik der Unterdrückten (Paulo Freire) den emanzipatorischen Erziehungsprojekten der nördlichen Wohlstandsregionen entgegen.

In den 1970er und 8oer Jahren setze dann eine die erste überlagernde, zweite Welle kritischer Erziehungswissenschaft ein, die sich am französischen Macht-Wissensdiskurs Michel Foucaults, am kriminologisch-sozialpädagogischen Labelling-Approach, an Goffmans Studien über Stigmatisierung und totale Institutionen orientierte, die zumeist in der einen oder anderen Weise auch Erziehungs-, Sozialisationsund Disziplinierungsagenturen waren (Foucault 1976/1969; Goffmann 1961/1963). Hinzu kam ein breiter Diskurs über die von Jean Piaget und Lawrence Kohlberg ausgehenden, von Rainer Döbert und Gertrud Nunner-Winkler auf deutsche Verhältnisse zugeschnittenen und kritisch weiterentwickelten Theorien der kognitiv-moralischen Sozialisation und Erziehung, die Hoffnungen auf einen kräftigen Schub postkonventioneller, also kritischer Grundorientierungen in spätkapitalistischen Gesellschaften weckten, zumal die breite Rezeption dieser Theorien mit der seit Beginn der 1960er Jahre explosionsar- 
tigen, bis heute anhaltenden globalen Expansion des Erziehungsund Bildungssystems, dadurch verlängerter Jugend etc. zusammenfiel (Döbert/Nunner-Winkler 1975; Garz 2006). Linke Politik, fast überall motiviert durch die globale Bewegung der Studenten, der Frauen, der Homosexuellen und der People of Color kam den emanzipatorischen, jetzt auch häufig antipädagogisch radikalisierten Tendenzen der Erziehungswissenschaft und Sozialpädagogik entgegen (von Braunmühl 1975; vgl. Rutschky 1977). Der Tunix-Kongress wurde 1978 zum Fanal einer neuen Praxis, die stark durch die französische Theorie, allen voran den Poststrukturalismus, bestimmt war. Später kamen Louis Althusser und seine Schüler, aber auch Jacques Lacan und Emmanuel Levinas hinzu, vom neuen gender-orientierten Feminismus und Judith Butler gingen weitere starke Impulse aus. Aber immer noch ging es um Kritik, ergänzt um Gegenwarts-Archäologie, fröhlichen Positivismus, unentrinnbare Performanz und das Spiel diskursiver Macht-Techniken. Die Kritik wurde immer reflexiver. Kritik der Kritik der Kritik. Ad infinitum immer radikaler und subtiler - bis hin zur Aufdeckung unserer postkolonial unaufgeklärten Mikroaggressionen. Aber letztlich folgt die Erziehungswissenschaft in ihrer jeweiligen Avantgarde der von Kant und Marx bis zu Max Horkheimers und Theodor W. Adornos Dialektik der Aufklärung (1944) reichenden Spur der Kritik. Selbst wenn sich die Spur des Menschen im Sand verlieren sollte, wie Foucault es erwartete, vielleicht auch von der Wirkung seiner und anderer posthumanistischer Schriften erhofft haben mag, die Kritik bleibt. Hochschuldidaktisch war es die Zeit der autonomen Tutorien. Nicht zufällig sah sich der späte Foucault denn auch in der Tradition Immanuel Kants und der französischen Aufklärung und in Übereinstimmung mit der von ihm spät rezipierten Dialektik der Aufklärung, von der er selbst sagte, eine frühere Lektüre hätte ihm viel Mühe erspart. Wichtig wurde auch die schon erwähnte Erweiterung des Kapitalbegriffs ins symbolische, soziale und ästhetische, mit der Bourdieu, an Claude Lévi-Strauss anschließend, aber nicht von ihm abhängig, weit über Marx hinausgeht. Vom Kapital bei Bourdieu war bald ähnlich ubiquitär die Rede wie von der Macht bei Foucault. Das eine mal war die Hauptstoßrichtung der Ausschluss derer ganz unten, 
das andere mal der Ausschluss derer am Rand, des infamen Menschen. Aber immer noch Kritik, die der Pädagogik ein weiteres hoffnungsvolles - und was wäre Pädagogik ohne Hoffnung? - Programm nahelegte: das der Vollinklusion.

Dann kam Niklas Luhmann und entfachte einen kurzen, heftigen Begeisterungssturm unter den Erziehungswissenschaftler*innen. Er sah die Dinge ähnlich wie Foucault, verzichtete aber auf die Autonomie im Widerstand und die Selbstpraktiken des Subjekts: "Das Subjekt ist kein Objekt, was soll es also in der Theorie!« (Luhmann 1973: 21; Alvear Moreno 2020). Das einmal eingesehen, war das Subjekt vom Alp, sich emanzipieren zu müssen, ein für alle Mal befreit: Die lebenslange Verurteilung zur Freiheit wurde von der höheren Instanz des Beobachters zweiter Ordnung aufgehoben und in einen sich selbst paradoxierenden Freispruch umgewandelt. Durch das System, das es ist, war das frühere (alteuropäische) Subjekt auf bestem Weg in den glücklichen Positivismus, den Foucault propagiert hat, aber nie erreichen wollte. Aus der Systemtheorie musste das frühere Subjekt jetzt nur noch lernen, das System, das es ist, so zu beschreiben, das es seine gesellschaftliche Funktion erfüllt, und: statt sich darein zu verzetteln, den unerkennbaren blinden Fleck im eigenen Bewusstseinssystem zu suchen, in nunmehr »abgeklärter Aufklärung« die Evolution machen zu lassen und ihr nicht ins Handwerk zu pfuschen. Zwar weiß die Evolution auch nicht, wo es langgeht, aber sie weiß doch besser als der besserwisserische Mensch, wie man den jeweils nächsten Schritt macht, ohne von der Überkomplexität der Umwelt sofort verschluckt zu werden (Luhmann 1974 (1970): 67). So auch in der Pädagogik. Sie ist unentrinnbar Macht-Technik, und ihre Rationalität lässt sich nur steigern, wenn man sich nicht wie Foucault trotzig auf Gegen-Macht versteift oder auf den verschlungenen Frankfurter Wegen ihrer technischen Vernunft $\mathrm{zu}$ entkommen sucht, sondern das System machen lässt, sich seines Technologiedefizits bewusst wird und diesem das kontrafaktische, aber funktionale, weil Komplexität reduzierende Festhalten seiner Interaktionssysteme (Lehrer, Schüler) an ihren ersichtlich falschen Kausalplänen empfiehlt und die Emanzipation dem naturwüchsigen, »unterschwelligen Chaos im Klassenzimmer« über- 
lässt. Das aber heißt nichts anderes, als das Resultat allen Unterrichts dem sozialen Selektionsmechanismus zu überlassen, der gleich am ersten Schultag damit beginnt, aus winzigen Unterschieden des Sitzfleisches, der Konzentrationsfähigkeit und der Intelligenz Riesenunterschiede $\mathrm{zu}$ machen. Das genau ist die unhintergehbare gesellschaftliche Rahmenbedingung: die Funktion der Schule und des gesamten Bildungssystems (Luhmann/Schorr 1979/1982: 11- 40; Luhmann 1992/93). Der Umbau der naiven, aber falschen Kausalpläne in den Köpfen von Lehrern und Schülern in reflexive »Technologieersatztechnologien«, die nicht weniger falsch sind, machen dem Lehrsystem ein für alle Mal klar, »[that] we have to come to terms, once and for all, with a society without human happiness and, of course, without taste, without solidarity, without similarity of living conditions (Luhmann/Schorr 1982; Luhmann 1997: 70). Aber auch das ist nur eine weitere, reflexive Schleife der Kritik der Kritik, die immer Kritik ist, weiß sie es doch besser, denn sie sieht, was du nicht siehst (Luhmann 1990: 228-234). Zwar soziologisch abgeklärt, aber immer noch Aufklärung. Diesmal sogar als »Hochform technokratischen Bewusstseins « (Foucault 1986: 178, 240; Habermas 1971: 145).

\section{II}

Aber all diese Versuche, Neuansätze, Radikalisierungen kritischer Pädagogik konnten - so mag es den angesichts der systemischen Selektionsmechanismen frustrierten Pädagogen nach Mollenhauer, Foucault, Tunix, Antipädagogik, Paulo Freire, Bourdieu und Luhmann erschienen sein - letztlich die Frage nicht beantworten, warum progressive Erziehung trotz der Vielfalt und Originalität ihrer vielen Varianten immer wieder scheitert?

Die Erfahrung dieses Scheiterns - »Ja mach nur einen Plan!/[...] Und mach dann noch 'nen zweiten Plan/Gehn tun sie beide nicht.« (Brecht) -, die Luhmann und Schorr in die pädagogischen Kausalpläne einbauen wollten, führen um die Jahrtausendwende und in den ersten beiden Jahrzehnten des 21. Jahrhunderts zur völligen Verdrängung der 
Utopie durch die Dystopie. Die pädagogischen Optimisten wurden vor das Gericht einer pessimistischen, mittlerweile spieltheoretisch, systemtheoretisch und poststrukturalistisch vergesellschafteten Sozialanthropologie gestellt, um, anders als in den 1960er Jahren, ihr Unvermögen kleinlaut einzugestehen (Habermas 1961: 251-278). Sie gestehen teils aus Einsicht, teils aus Anpassung, dass ihnen misslungen sei, das Unvernehmen vernehmlich zu machen. Sie bezichtigen sich selbst, die polizeiliche nur durch eine emanzipatorische Vernehmung substituiert zu haben, die lediglich eine subtilere Form der exkludierenden Unterdrückung des Unvernehmens ist (Rancière 2002). Kritische Pädagogik, emanzipatorische Erziehung, Antipädagogik, Tunix, Selbsttechnik, Vollinklusion - immer wieder endete der politische wie der pädagogische Versuch, das Unvernehmen in emanzipatorischer Absicht vernehmlich zu machen, in einer neuen Vernehmung.

Das war, so die Diagnose der postkritischen Pädagogik, eine Folge der Bevormundung durch eine Kritik, die Kritikfähigkeit, Emanzipation, Mündigkeit, Autonomie durch pädagogischen Interventionismus, also letztlich durch technische Herrschaft über Erziehungs- und Bildungsprozesse erreichen wollte (Hodgson et al. 2022: 23). Darauf zielen die drei Einwände, also die (Post-)Kritik, mit der das Manifest die kritische Pädagogik konfrontiert. Dem Educandus, -a, -um werde von der kritischen Pädagogik 1. ein Mangel an kritischem Bewusstsein unterstellt, der sie*ihn hindere, die Ideologie im eigenen Kopf zu durchschauen, wodurch 2. die Ungleichheit, also die Klassengesellschaft im pädagogischen Mikrokosmos symbolisch reproduziert werde - ob die Lehrer-/Erzieher*innen es nun wissen oder nicht, "sie tun es« (vgl. Marx 1969: 88) und sie tun es, um 3. den latenten Hass der schlechter Weggekommenen gegen die besser Weggekommenen manifest $\mathrm{zu}$ machen und in die richtige Richtung zu lenken. ${ }^{1}$ Auch noch die Techniken der Gegenmacht, der Lebenskunst, der Kultur seiner selbst,

$1 \quad$ Nur mit sehr viel Nachsicht - principle of charity - kann man diese Kritik der Kritik als Kritik der Kritik der Vernunft (Kant), der politischen Ökonomie (Marx) oder der Frankfurter kritischen Theorie wiedererkennen, eher schon den Leninismus, zu dem sich die genannten drei Kritiken ihrerseits kritisch verhalten. 
der experimentellen Selbstpraktiken und der zugehörigen Hermeneutik des Subjekts (Foucault 1985), der Sorge um sich und den eigenen Körper, des »Horchens auf eine Vernunft, die der natürlichen Ordnung« vorausgeht und auf die "gesetzesfreie Allgemeinheit einer Ästhetik der Existenz« gerichtet ist, bleiben als Reflexion, Kritik und Selbstkritik bei aller Gesetzlosigkeit eine weitere Hochform technokratischen Bewusstseins. Evident wird das in der (neoliberal anschluss- und globalisierungsfähigen) Substitution des soeben als herrschaftsfunktional entlarvten Sprachspiel der Emanzipation durch das keineswegs minder herrschaftsfunktionale des Empowerment (Somek 2013: 258-282). ${ }^{2}$ Was tun, nachdem weder Kritik noch die vervielfachte und diversifizierte Kritik der Kritik, weder Emanzipation noch Empowerment, weder Tun noch Nichts-Tun die Herrschaftsverhältnisse dieser Welt in Politik und Pädagogik grundstürzend erschüttert haben? Und was wäre Pädagogik ohne Hoffnung auf Weltveränderung, also ohne die 11. Feuerbachthese, die ja auch das Credo der postkritischen Pädagogik und einer ihrer Heldinnen, Hannah Arendts, ist?

In diesem Augenblick der Krise der Kritik trat Rancière an die Stelle von Kant, Marx, Adorno, Foucault, Bourdieu etc. Nur, was sich so spontan vollzieht wie der große Aufstand der Pariser Unterschichten im Juni 1848, kann sich überhaupt von Herrschaft, Heteronomie und Unmündigkeit befreien, weil nur so ein Aufstand, weil nur so eine Politik, weil nur so eine Pädagogik frei ist von neuer Bevormundung, Besserwisserei und der Autorität wohlwollender Kritiker, enthusiasmierter Befreier, Emanzipateure, Fortschrittler etc. (vgl. Rancière 1991). Mit ihren Barrikaden verwandeln die Aufständischen, so Rancière, die »städtischen Frage: „Queerfeminismus befindet sich - in Zeiten in denen Deutsche Bank, Burger King und Ford einmal im Jahr spontan queer werden - zwischen gesellschaftlicher Anerkennung und Vereinnahmung. Was bedeutet es, wenn wir von Empowerment statt Emanzipation, von Beyoncé statt Audre Lorde sprechen, davon soziale Hierachien nicht abzuschaffen sondern zu diversifizieren. Bedarf es wirklich nur eines \#CirlBoss um unsere Welt zu verändern?« https://b ewegungsmelder-aachen.de/events/queer-feminism-for-sale/ (5.9.2021). 
Verkehrswege buchstäblich in öffentlichen Raum« und machen sichtbar, »was keinen Ort hatte, gesehen zu werden « (ebd.: 41; Sartre 1967: 273-280/389-388). ${ }^{3}$ Das ist das Paradigma der postkritischen Pädagogik. Indem sie durch ihre spontane, von keiner kritisch geschulten Parteipolizei angeführte, politische Aktion die offizielle Politik als polizeiliche Ordnung der Ein- und Ausgrenzung kenntlich, sinnlich sicht- und hörbar machen, verwandeln sie die bis dahin unpolitischen Klassengegensätze in einen politischen Streit jenseits von Partei und Polizei. Das ist das Fanal, das »Geschichtszeichen « des Juni 1848, eines exemplarischen, aber erfolglosen, von der Polizei blutig geschlagenen Aufstands - »über 3000 Insurgenten wurden niedergemetzelt nach dem Siege, 15.000 ohne Urteil transportiert «(Marx 1985: 105) ${ }^{4}$ Aber so ein Aufstand »vergisst sich nicht « und bleibt als »wiederholbares« Muster im kulturellen Gedächtnis der Gesellschaft haften: als Contra-Erinnerung, in Zukunft die Gerechtigkeit nicht zu vergessen (Kant 1977: 357-361; Assmann o.J.: 103; Boehm 2020: 89,42,59ff.). ${ }^{5}$ Damit wäre die Geburt der postkritischen Pädagogik geschafft. Was bleibt, sind die Mühen der Ebene.

III

Die Ebene beginnt mit einem Manifest und fünf Grundsätzen. Manifeste sind spätestens seit Februar 1848, als das von Marx und Engels verfasste Manifest der kommunistischen Partei in London auf Deutsch erscheint, der schriftlicher Ausdruck »revolutionärer, praktisch-kritischer Tätigkeit«, in denen eine Avantgarde sich selbst zur Spitze des Fortschritts der Gesellschaft, der Kunst oder der Wissenschaft erklärt,

3 Mit Sartre könnte man auch von der spontanen Transformation einer seriellen in eine fusionierende Cruppe - eine Art sozialer Klasse für sich - sprechen.

4 Mit »transportieren « meint Marx die Deportation auf eine lateinamerikanische Gefangeneninsel im Südatlantik.

5 Zur Contra-Erinnerung: Jan Assmann; Zur prophetischen Erinnerung: Omri Boehm. 
»ihre Anschauungsweisen, ihre Zwecke, ihre Tendenzen vor der ganzen Welt offen darlegt«, die Aufhebung und Zerstörung einer »alt gewordenen Gestalt des Lebens« ankündigt und die Geburt einer »neuen Zeit « und eines neuen Bundes - sei es eines Gottesbundes, sei es eines Bundes der Kommunisten - nicht prognostisch vorhersagt, sondern prophetisch, praktisch intervenierend »hervorsagt « (Marx/Engels 1990: 461; Hegel 1970: 28; Koch 1995: 256). ${ }^{6}$ Statt sie abzuwarten, ruft das Manifest »die neue Zeit« aus, »vorwärts lebend« in eine offene und unbekannte Zukunft: »Die Proletarier haben nichts zu verlieren als ihre Ketten. Sie haben eine Welt zu gewinnen. Proletarier aller Länder, vereinigt Euch!« (Hegel 1970: 491; Kierkegaard 1941: 162; Marx/Engels 1990: 493). ${ }^{7}$ Eine Utopie, aber dem Anspruch nach eine reale.

Diesmal aber, 169 Jahre später, im Oktober 2017 ist das Manifest ein Manifest für eine postkritische Pädagogik. Das lässt auf den ersten Blick weder kritische Theorie und Praxis, noch Avantgarde und Fortschritt, noch die Zerstörung einer alten und die Vorhersage einer neuen Gestalt des Lebens erwarten (vgl. Tyson/Lewis 2017: 23-34). Also realpolitische Dystopie?

Das Manifest erklärt sich in fünf Grundsätzen, unter denen die Autoren keine platonischen Ideen, sondern wie Marx und Engels die Anschauungen, Zwecke, Tendenzen pädagogischer - und wohl auch politischer - Post-Kritik verstehen. Aber die Erwartung, die Post-Kritik als subjektlose Evolution, als Polizei ohne Politik auf der funktionalistischen Seite (Luhmann), oder aber als gesellschaftslose Praxis, als Politik ohne Polizei auf der spontaneistischen Seite (Rancière) zu verstehen, wird sofort enttäuscht.

Der erste Grundsatz übers Prinzipielle grenzt sich von der Postmoderne $a b$, indem er an universellen Prinzipien festhält, aber den $\mathrm{Ne}$ gativismus der Kritik überwinden möchte. Stattdessen verlangt er, in affirmativer Attitüde nach Hoffnungszeichen in der bestehenden pädagogischen Praxis - eine »affirmative Haltung « unter »aktuellen Bedingungen « - zu suchen (Hodgson et al. 2022: 20). Das wollte Kant auch,

6 In der Reihenfolge der Zitate.

7 In der Reihenfolge der Zitate. 
als er glaubte, in der Französischen Revolution habe sich ein wirklicher normativer Fortschritt ereignet, in dem er ein hoffnungsvolles Geschichtszeichen der beginnenden Verwirklichung der Vernunft gesehen hat. Aber hier bezieht sich das Moment der Affirmation auf ein winziges Moment der damaligen Wirklichkeit, und das auch in erster Linie als bleibende reale Möglichkeit gegenwärtiger Zukunft. Nicht anders das kommunistische Manifest, das solche Hoffnung in der beginnenden Vereinigung der Arbeiter in Gewerkschaften und politischen Parteien zu erkennen glaubte - die zugehörigen Lernprozesse emanzipatorischer politischer Pädagogik eingeschlossen. Es geht also weder im postkritischen Manifesto noch bei Kant und Marx um platonische Ideen oder Ideale (»nicht Normativität [...] eines Ideals eines gegenwärtigen oder zukünftigen Zustands, an dem die gegenwärtigen Praktiken beurteilt werden sollen« (ebd.: 20)), sondern um deren gesellschaftliche Wirklichkeit, die nicht auf ein feststehendes Telos zuläuft, sondern in praktisch-kritischer Tätigkeit vorwärts lebend gemacht werden muss. ${ }^{8}$ Andererseits soll die Normativität solcher Hoffnung nicht »prozeduraler« - also machbar und juristisch (polizeilich) anschlussfähig -, sondern prinzipieller Natur sein: grundsätzliche Normativität (»prinzipientreue[] Normativität«) (ebd.: 20) Was das heißt, scheint der zweite, weniger progressivistische Grundsatz zu erklären.

Im zweiten Grundsatz lehnen die Autoren des Manifesto den Weg von Marx und Engels ab, den Klassenkampf - oder strukturelle Konflikte - ins Zentrum post-kritischer Pädagogik zu rücken und setzen stattdessen auf pädagogische Hermeneutik und Gemeinsamkeit: »[...] trotz vieler Differenzen, welche uns voneinander trennen, einen Raum der Gemeinsamkeiten gibt, der sich ausschließlich aus der Erfahrung (a posteriori) ergibt « (ebd.: 21). Das, so scheint es, kommt Gadamers »Rehabilitierung von Autorität und Tradition« näher als dem Vorschlag

Vgl. Kierkegaard 2003: 318: Dort heißt es über eine Vorlesung Schellings im WS 1843 in Berlin unter Anwesenheit fast aller Junghegelianer: »Ich bin so froh, Schellings zweite Stunde gehört zu haben - unbeschreiblich. So habe ich denn lange genug geseufzt und haben die Gedanken in mir geseufzt; als er das Wort Wirklichkeit nannte.« 
von Marx, den "Alp aller toten Geschlechter, der auf dem Gehirne der Lebenden lastet«, abzuschütteln, um die »eigene Geschichte [...] unter selbst gewählten Umständen« zu machen (Gadamer 1975: 261268; Marx: 96f.). Damit schloss Marx unmittelbar an die Idee einer demokratischen Verfassung der Gesellschaft an, die spätestens seit den atlantischen Revolutionen des späten 18. Jahrhunderts (Haiti, Nordamerika, Frankreich) den politischen und juristischen Diskurs beherrschte und der entgegenstehenden Verfassungsrealität überall widersprach. Verfassung heißt seitdem, einen reflexiven Schritt zurückzutreten, um die Umstände, unter denen »die Menschen« ihre eigene Geschichte machen, aus "freien Stücken« selbst $\mathrm{zu}$ wählen (Marx: 96f.). Genau das ist Autonomie. Jedenfalls für Kant, der diesen Begriff in die Philosophie eingeführt hat. Kant hat sie als eigene und dennoch allgemeine Gesetzgebung bestimmt, und zwar sowohl in der Moral wie im Staatsrecht (Kant 1968/1977: 65,432; vgl. Tugendhat 2007: 129).

Der dritte Grundsatz zieht jedoch der pädagogischen Hermeneutik postwendend den konservativen Zahn, um sie mit Rancières "Annahme der Gleichheit « zu egalisieren, und darin sehen die Autoren des Manifests den eigentlichen Wechsel von der kritischen zur postkritischen Pädagogik, um sich sogleich zu versichern, das sei aber in keiner Weise anti-, sondern eben postkritisch (Hodgson et al. 2022: 21). Das 20. Jahrhundert hat uns zwar mit einem immer noch nützlichen, »enormen und äußerst mächtigen kritischen Apparat[]« ausgestattet, aber die immer tiefer dringende, entlarvende Kritik hat seine Kritiker die affirmativen Aufgaben, zu »schützen und zu sorgen (vgl. Latour, Haraway)«, vergessen lassen (ebd.: 20). Statt sie als herrschaftsfunktionale Einrichtungen i. S. einer Hermeneutik des Verdachts zu entlarven, sollten wir i. S. einer Hermeneutik des Subjekts wieder darauf achten, was Erziehung, Aufwachsen, Schule und Schulung, Studieren, Denken und Praktizieren eigentlich sind und ihnen einen ermöglichenden Rahmen (Czejkowska 2022) schaffen, das zu werden, was sie von sich aus sein wollen. Prinzipielle Normativität soll dafür sorgen, dass sie das auch können.

Wofür sorgt eine solche prinzipientreue Normativität? Wie Agnieszka Czejkowska in ihrem Beitrag zu diesem Band m.E. wohl 
zurecht vermutet, geht es den Autoren des Manifests »durchwegs klassisch um die Klärung der Bedingungen der Möglichkeit, Aussagen über Erziehung treffen zu können «, und das "umfasst eine wissenschaftliche Fundierung pädagogischer Prozesse und den so wichtigen Bereich der Kritik institutioneller Rahmenbedingungen dieser Prozesse« (Czejkowska 2022: 167). Principled normativity sorgt also durch die Kritik und Bevormundung nicht des Educandus, -a, -um, sondern der institutionellen Rahmenbedingungen, in die er*sie eingezwängt ist, dafür, dass er*sie sich spontan so verhalten so verhalten kann, wie er"sie ist und sein will und - das ist entscheidend - sich ihren"seinen »eigenen und dennoch allgemeinen « (Kant) und deshalb gesetzgebenden Willen bilden kann. Das ist politische Bildung at its best. Sie kritisiert und bevormundet niemanden, ermöglicht, wenn die Kritik der Rahmenbedingungen praktisch-tätig erfolgreich ist, die egalitäre Kritik, Bevormundung und praktisch-tätige Erzeugung (Revision) der institutionellen Rahmenbedingungen durch die, an die ihre Spielregeln und Normen adressiert sind: die pädagogische Community als Realisierung von Autonomie.

Das aber impliziert, dass die kritische nicht in der postkritischen Pädagogik verschwindet, sondern genau auf die höhere Stufe verschoben (aufgehoben) wird, wo Kant und Marx den modernen Begriff der Kritik, und ihnen folgend, die kritische Theorie (Frankfurter Schule) sie immer von vornherein lokalisiert haben: auf der reflexiven Stufe der Bedingungen der Möglichkeit von Erkenntnis und Praxis, also auch Erziehung und Sozialisation. Und nur auf dieser, auf die Totalität der Gesellschaft zugreifenden Stufe kann Kritik überhaupt wirksam, radikal, progressiv, revolutionär sein. Aber nicht mehr, wie bei Kant, wenn wir ihn neukantianisch (und damit wahrscheinlich falsch) verstehen, als transzendentale Kritik von einem gesellschaftsexternen Gottesgesichtspunkt (Putnam) oder View from Nowhere (Nagel) aus, sondern wie bei Marx als Gesellschaftstheorie mittenmang: im "Handgemenge« (Marx 1972: 381).

Der vierte Grundsatz postuliert einen Wechsel der um der Erziehung willen (ihrer Autonomie also) nunmehr nachhaltig dekonstruierten instrumentellen Erziehung des grausamen Optimismus, des »cruel optimism« der Vorstandsvorsitzenden, Generäle und Realpolitiker zuguns- 
ten gegenwärtiger Hoffnung - »hope in the present« - auf eine bessere Zukunft in einer globalen Rechtsgenossenschaft, die man sich am besten mit Emerson und Rorty als linkes Projekt der »Partei der Hoffnung« vorstellt (Hodgson et al. 2022: 23; Rorty/Vetter 1999: 19). Das ist die Partei der »gesellschaftlichen Hoffnung auf eine »Great Community« (Dewey) »ohne Kasten, ohne Klassen, ohne Grausamkeit, « zu dem Zweck geschaffen, »to expand the reference of us as far as we can, but of us at our best « (Rorty 1999: XIII-XIV; Rorty 1993: 452). Diese große Gemeinschaft hat Dewey immer als große, demokratische Gemeinschaft der lehrend Lernenden und lernend Lehrenden verstanden, also als vollständig demokratisierte Universität - ein Traum, der in den 1960er Jahren von Berkeley aus um die Welt ging, bevor er überall von der Polizei (Rancière) blockiert wurde. Eine solche Partei der Hoffnung könnte sich ohne Bevormundung ruhig als »Vorhut der menschlichen Geschichte« verstehen, in deren Praxis sich »die Verheißung aller Zeiten zuerst erfüllt« und deren »Wesen« »unsere Existenz« ist, die »in der Zukunft liegt« (Rorty 1998: 19,23,15,27). Eine solche Existenz, die alle »bestehenden Bezugssysteme sprengt « - nicht das imperiale, sondern Rortys utopisches Amerika, das es überall gibt - muss sich »die Maßstäbe erst schaffen, nach denen sie beurteilt« wird (ebd.: 33). Das ist die gleichzeitig politische, gesellschaftliche und pädagogische Hoffnung, die der vierte Grundsatz einer - und ich würde spätestens jetzt den Ausdruck "postkritisch « fallenlassen, weil er genauso unglücklich und missverständlich ist wie derjenige der "Postmoderne« - kritischen Pädagogik, die sich auf die höherstufige Kritik der institutionellen Rahmenbedingungen möglicher Erziehung (ganz i. S. von Kants grenzziehendem Gebrauch der Worte »Kritik« und »transzendental«) beschränkt und sich direkt auf den fünften Grundsatz der Liebe zur Welt bezieht, der »love for the world«: Der Liebe zur Welt, die alle in dem Augenblick zu gewinnen haben, wenn sie ihre sichtbaren und unsichtbaren Fesseln verlieren. 
Was heißt hier Liebe (love for the world)? - Der Begriff, so wie ihn das Manifest der Postkritik verwendet, hat religiöse Konnotationen, die auf das Neue Testament, aber auch auf alle anderen Weltreligionen der Achsenzeit verweisen.

In seinem Plädoyer für die Vereinigung der alten Linken, die sich der sozialen Gleichheit und der Emanzipation von Klassenherrschaft verschrieben hat, mit der neuen Linken, deren Ziel die Befreiung von Stigmatisierung, Sadismus und Diskriminierung ist, hat Richard Rorty zwei Bücher für die Lektüre der jungen Generation vorgeschlagen: das Neue Testament der Liebe und das Kommunistische Manifest des Klassenkampfes. Das Neue Testament wegen dessen unüberbietbarer Darstellung der »Sehnsucht nach Brüderlichkeit« und das Kommunistischen Manifest wegen seiner »klaren Einsichten in die neuesten Formen unserer Unmenschlichkeit im Umgang miteinander«, die ihre Leser »inspirieren und ermutigen, « sich - mit Kant - ihres eigenen Verstandes zu bedienen, um sich aus »selbstverschuldeter Unmündigkeit« zu emanzipieren (Rorty 1998: 11,29; Kant 1977 : 53).

Der Mensch, der lernt, sich aus selbstverschuldeter Unmündigkeit $\mathrm{zu}$ emanzipieren, ist ein Subjekt »ohne anthropologische [oder gattungsspezifische] Konstanten, « das, wie Rousseau schon gesehen hat, nicht nicht lernen kann (Geyer 1997: 210; Rousseau 1984: 79-85; Greenwood 2015). Außer biologischen Konstanten, die es mit allen Säugetieren teilt, ist das menschliche Subjekt ein durch und durch gesellschaftliches Wesen, und auch seine biologischen Konstanten sind seit langem durch Sozialisationsprozesse epigenetisch überformt und verändert, mittlerweile durch Artefakte jeder Art ergänzt, modifiziert, substituiert (vgl. Jalonka/Lamb 2014; Moore 2015). Kurz: Der Mensch ist ein »Ensemble der gesellschaftlichen Verhältnisse«, ein Wesen, das sich nur in der Gesellschaft und durch Gesellschaft subjektivieren kann, in der es sich als jemand erfährt, der*die für sein durch und durch gesellschaftliches Schicksal selbst verantwortlich ist, weil sie ihre »eigene Geschichte machen « (Marx 1993/1955/1985: 6, 6, 96f.). Auf das Machen kommt es an. Denn das Machen der eigenen Geschichte erlaubt den 
immer schon positiv oder negativ (konfligierend) kooperierenden Akteuren den stets gegenwärtigen (»present«), konstituierenden, also weltverändernden und befreienden Zugriff auf die »unmittelbar vorhandenen, gegebenen und überlieferten « Umstände, denen sie unterworfen sind und die »wie ein Alp« auf ihrem Hirn lasten (Marx 1985: 96f.). Das Subjekt ist auch noch als machtunterworfener, versklavter und infamer Körper frei, »Nein« zu sagen und sich im äußersten Fall undienlich zu machen (Dämann 2020; vgl. Habermas 1981: 445-452). ${ }^{9}$ Das ist auch die auf Kant, Marx, Därmann, Adorno, Sartres Folteranalyse, Habermas, Rancière usw. zurückgehende Prämisse des postkritischen Manifestos: "At the very heart of a relationship that defines inequality (slavery), Rancière finds a disavowed reliance upon the equality of intelligences; for how can the slave carry out the master's orders if he or she is not already capable of thinking and speaking? « (Lewis 2017: 30; vgl. Rancière 2004). ${ }^{10}$ Kooperation ist für ein Wesen, das "nur in der Gesellschaft sich vereinzeln kann «, unvermeidlich (Marx 1955: 6). Also ist das Subjekt immer auch deshalb machtunterworfen, weil es trotz aller falschen und repressiven »Seelsorger«, »Ärzte«, »Oberaufsichten«, »Gängelwägen« und »Fußschellen«, die es einzwängen, überwachen, unterdrücken, strafen, beherrschen letztlich doch als kooperativ handelndes Subjekt, also selbstverschuldet in den Stand, den Staat, die Verfassung, kurz: die institutionellen Rahmenbedingungen der Unmündigkeit geraten ist, die es eben deshalb nur selbst ändern und in eine egalitäre Verfassung der eigenen und dennoch allgemeinen, nämlich »mitgenossenschaftlichen« Selbstgesetzgebung verwandeln kann (Kant XI: 53f.; Kant 1977: 424). Das Subjekt selbst hat aus »Erziehung, Tradition, Gewohnheit die Anforderungen« des Kapitals oder der Macht - also naturwüchsige gesellschaftliche Zwangsverhältnisse - »als selbstverständliche Naturgesetze anerkannt (Marx 1969: 765). Anerkennen aber kann man nur, was man auch ablehnen kann. Die "schicksalsvollste Macht unseres modernen Lebens: der Kapitalismus« (Weber), ist zwar ein ursächlich herrschaftlich erzwungenes, objektives »Abhängigkeitsverhältnis«, das aber ohne ein

9 So zuletzt: Iris Därmann.

10 So zutreffend Lewis/vgl. Rancière. 
änderbares, reziprok entgegenkommendes, subjektives »Abhängigkeitsgefühl« (Marx) nicht existieren kann (Weber 1978: 4). Dieses Gefühl erzeugt Mutlosigkeit, die aber durch den »Mut, «sich seines »eigenen Verstandes zu bedienen " (Kant), aufgehoben werden kann, um als Subjekt der eigenen Geschichte »die Tradition aller toten Geschlechter, « die "wie ein Alp auf dem Gehirne der Lebenden lastet«, abzuschütteln (Marx 1969: 797; Kant 1977: 53; Marx 1985: 97). Nur deshalb kann das vermeintlich postkritische Manifesto behaupten: »[...] pädagogische Hoffnung richtet sich an die Möglichkeit der Erneuerung unserer gemeinsamen Welt« (Hodgson et al. 2022: 23). Nicht aus den Evangelien, wohl aber aus dem Kommunistischen Manifest können die menschlichen Subjekte die für solchen Mut »wichtige Lektion« über den modernen Kapitalismus ohne Bevormundung, sondern einfach durch Lesen und Diskutieren lernen, »dass der Sturz autoritärer Regierungen und die Schaffung konstitutioneller Demokratien zur Sicherung von Gleichheit und Anstand zwischen den Menschen nicht ausreichen« (Rorty 1998: 20). Und dass wir bei aller Liebe zur Welt die "Wahrheit« nicht länger verleugnen sollten, dass »alle Geschichte immer noch die Geschichte von Klassenkämpfen« und der moderne Staat nur ein Ausschuss ist, »der die gemeinschaftlichen Interessen der ganzen Bourgeoisklasse verwaltet« (ebd.: 2of.). Schließlich könnten die lernenden Akteure unter günstigen Rahmenbedingungen durch Lektüre und Diskussion selbst erkennen, dass »das Manifest « sich wegen seiner soziologischen Einsichten und seiner moralischen Schärfe »doch noch besser als die Evangelien zur Lektüre für die Junge Generation« eignet. Vergleichend könnten sie lernen, dass dem Manifest die »erheblichen moralischen Unschärfen« fehlen, die das Evangelium »aufgrund seiner Jenseitsorientierung « aufweist, weil »viele Passagen Sklavenhaltern den Eindruck vermitteln", "sie könnten getrost weiter die Peitsche schwingen," während andere Passagen »Reiche Glauben machen, sie dürften die Armen getrost weiter hungern lassen. In den Himmel kämen sie trotzdem, denn ihre Sünden würden ihnen vergeben, da sie ja Christus als ihren Herrn anerkannt hätten« (ebd.: 25f.). Schließlich könnten sie ohne jede Bevormundung selber lernen, aus der Tatsache der stalinistischen und inquisitorischen Verbrechen und der bürokratischen 
Herrschaft sowjetischer Nomenklaturen und katholischer Kleriker nicht den Fehlschluss zu ziehen, die Inspirationskraft des Evangeliums und des Kommunistischen Manifests würden »dadurch geschmälert, dass Millionen Menschen von aufrichtigen, moralisch gefestigten Leuten versklavt, gefoltert, in den Hungertod getrieben wurden, die sich zur Rechtfertigung ihrer Taten auf Abschnitte aus einem dieser beiden Texte beriefen« (ebd.: 16).

Nichts weiter müsste eine kritische Pädagogik tun, wenn sie ihre Kritik nicht auf die Lernenden, zu denen sie selbst gehört, sondern auf die institutionellen Rahmenbedingungen möglicher Lernprozesse richtet. Dazu aber würde auch eine Gesetzgebung - also Rancières Polizei - gehören, die den marktkonformen Zustand eines Bildungssystems überwindet, das aufgrund seiner Selektionsfunktion dafür sorgt, dass "many great scientific minds, capable of contributing to the search for a COVID-19 vaccine or a strategy to climate-change, are languishing in slums« (Haider 2020). Genau diese Form der epigenetischen, normativen Beschränkung der evolutionären Mechanismen kommunikativer Variation und sozialer Selektion durch Märkte und Klasseninteressen, die es ermöglicht, das pädagogische Potential der Massendemokratie auszuschöpfen, hat Dewey unter demokratischer Erziehung verstanden. Es wäre die wichtigste institutionelle Rahmenbedingung, die erfüllt sein muss, um autonome Lernprozesse zu ermöglichen.

\section{Literatur}

Alvear Moreno, Rafael (2020): Soziologie ohne Mensch? Umrisse einer soziologischen Anthropologie, Bielefeld: transcript.

Assmann, Jan (o.J.): Politische Theologie zwischen Ägypten und Israel, München: Siemens- Stiftung: 103.

Boehm, Omri (2020): Israel - eine Utopie. Berlin: Ullstein.

Braunmühl, Ekkehard (1975): Antipädagogik - Studien zur Abschaffung der Erziehung. Weinheim: Beltz.

Czejkowska, Agnieszka (2022): »Tausende von Folianten stürzten mir auf den Kopf«, in: Martin Bittner, Anke Wischmann (Hg.): Kritik 
und Post-Kritik. Zur deutschsprachigen Rezeption des "Manifests für eine Post-Kritische Pädagogik«. Bielefeld: transcipt Verlag: S. 161-174.

Dahmer, Ilse; Klafki, Wolfgang (1968): Geisteswissenschaftliche Pädagogik am Ende ihrer Epoche, Weinheim: Beltz.

»Das Flugblatt des Weiberrats Frankfurt (1980)«, in: Miermeister, Jürgen/Staadt Jochen (Hg.): Provokationen, Neuwied: Luchterhand: S. $223 \mathrm{f}$.

Därmann, Iris (2020): Undienlichkeit. Gewaltgeschichte und politische Philosophie, Berlin: Matthes \& Seitz.

Foucault, Michel (1985): »Hermeneutik des Subjekts«, in: Foucault: Freiheit und Selbstsorge. Frankfurt: Materialis.

Foucault, Michel (1976): Überwachen und Strafen. Die Geburt des Gefängnisses, Frankfurt a.M.: Suhrkamp.

Foucault, Michel (1969): Wahnsinn und Gesellschaft. Eine Geschichte des Wahns im Zeitalter der Vernunft, Frankfurt a.M.: Suhrkamp.

Foucault, Michel (1986): Die Sorge um sich. Sexualität und Wahrheit 3, Frankfurt a.M.: Suhrkamp.

Gadamer, Hans- Georg (1975/1960): Wahrheit und Methode, Tübingen: Mohr.

Geyer, Paul (1997): Die Entdeckung des modernen Subjekts. Anthropologie von Descartes bis Rousseau, Tübingen: Niemeyer.

Goffman, Erving (1961): Asylums: Essays on the social situation of mental patients and other inmates, New York: Anchor.

Goffman, Erving (1963): Stigma. Notes on the management of spoiled identity, Englewood- Cliffs: Prentice-Hall.

Greenwood, Jennifer (2015): Becoming Human - The Ontogenesis, Metaphysics and Expression of Human Emotionality, Cambridge MA: MIT-Press.

Habermas, Jürgen (1961): »Pädagogischer Optimismus vor Gericht einer pessimistischen Anthropologie«, in: Neue Sammlung 1: S. 251-278.

Habermas, Jürgen (1964): »Von der Schwierigkeit, Nein zu sagen« in: Philosophisch-politische Profile. Frankfurt a.M.: Suhrkamp: S. 445452. 
Habermas, Jürgen (1971): »Theorie der Gesellschaft oder Sozialtechnologie?«, in: Jürgen Habermas, Niklas Luhmann: Theorie der Gesellschaft oder Sozialtechnologie - Was leistetSystemforschung?, Frankfurt a.M.: Suhrkamp: S. 142-290.

Haider, Asad (2020): The Use of Free Speech in Society, 15. July 2020 comments, https://www.vesobooks.com/blogs/4793-the-use-of-fre e-speech-in-society (6.9.2021).

Hegel, G.W.F. (1970): Grundlinien der Philosophie des Rechts, Frankfurt a.M.: Suhrkamp.

Hegel, G.W.F. (1970): Philosophie der Geschichte, Werke 12, Frankfurt a.M.: Suhrkamp.

Heydorn, Heinz- Joachim (1995): »Über den Widerspruch von Bildung und Herrschaft «, in: Ders.: Werke, Bd. 3, Vaduz: Topos.

Hodgson, Naomi; Vlieghe, Joris; Zamojski, Piotr (2017): »Manifesto for a Post-Critical Pedagogy«, in: Dies. (Hg.): Manifesto for a PostCritical Pedagogy. Earth: punctum.

Hodgson, Naomi; Vlieghe, Joris; Zamojski, Piotr (2022): »Manifest für eine Post-Kritische Pädagogik«, in: Martin Bittner, Anke Wischmann (Hg.): Kritik und Post-Kritik. Zur deutschsprachigen Rezeption des »Manifests für eine Post-Kritische Pädagogik«. Bielefeld: transcipt Verlag: S. 19-24.

Jablonka, Eva; Lamb, Marion J. (2014): Evolution in Four Dimensions - Genetic, Epigenetic, Behavioral, and Symbolic Variation, Cambridge MA: MIT-Press.

Kant, Immanuel (1968): »Grundlegung zur Metaphysik der Sitten« in: Werke VII, Frankfurt a.M.: Suhrkamp.

Kant, Immanuel (1977): »Der Streit der Fakultäten«, in: Ders.: Werke XI, Frankfurt a.M.: Suhrkamp, S. 357-361.

Kant, Immanuel (1977): Metaphysik der Sitten, Frankfurt a.M.: Suhrkamp.

Kant, Immanuel (1977): »Beantwortung der Frage: Was ist Aufklärung«, in: Werke XI in 12 Bänden herausgegeben von Wilhelm Wieschedel, Frankfurt a.M.: Suhrkamp: 50-61.

Kierkegaard, Sören (1941): Tagebücher 1834-1855, Leipzig: Hegner. 
Kierkegaard, Sören (2003): Die Tagebücher, Bd. 1, Grevenberg: Simmerath.

Koch, Klaus (1995): Propheten I, Stuttgart: Kohlhammer.

Lewis, Tyson E. (2017): »A Response to the »Manifesto for A Post-Critical Pedagogy«, in: Naomi Hodgson, Joris Vlieghe, Piotr Zamojski (Hg.): Manifesto for a Post-Critical Pedagogy, Earth: punctum: 23-34.

Luhmann, Niklas (1973): »Selbstthematisierung des Gesellschaftssystems« in: Zeitschrift für Soziologie 1: 55-66.

Luhmann, Niklas (1974): »Soziologische Aufklärung« in: Soziologische Aufklärung I, Opladen: Westdeutscher Verlag.

Luhmann, Niklas; Schorr, Karl-Eberhard (1979): Reflexionsprobleme im Erziehungssystem, Stuttgart: Klett.

Luhmann, Niklas; Schorr, Karl-Eberhard (1982): »Das Technologiedefizit der Erziehung und die Pädagogik «, in: Luhmann/Schorr (Hg.): Technologie und Selbstreferenz. Frankfurt a.M.: Suhrkamp: 11-40.

Luhmann, Niklas (1992/93): Theorie der Gesellschaft, Vorlesungsmitschnitt WS Uni Bielefeld, Carl-Auer Systeme Verlag.

Luhmann, Niklas (1997): »Globalization or World Society: How to Conceive of Modern Society?«, in: International Review of Sociology 1/1997: 67-79.

Luhmann, Niklas (1990): »Ich sehe was, was Du nicht siehst«, in: Soziologische Aufklärung 5, Wiesbaden: S. 228-234.

Mann, Thomas (2001): Betrachtungen eines Unpolitischen, Frankfurt: Fischer.

Marx, Karl (1969): Das Kapital I, Berlin: Dietz.

Marx, Karl (1985): »Der 18. Brumaire des Louis Bonaparte« in: MEGA I/11, Berlin: Dietz, S. 96-189.

Marx, Karl (1972): Kritik der Hegelschen Rechtsphilosophie. Einleitung, MEW 1, Berlin: Dietz.

Marx, Karl (1993): »Thesen über Feuerbach« in: MEW 3, Berlin: Dietz.

Marx, Karl (1955): Grundrisse der Kritik der politschen Ökonomie, Berlin: Dietz.

Marx, Karl/Engels, Friedrich (1990): »Manifest der kommunistischen Partei« in: MEW 4, Berlin: Dietz: 459-493. 
Mollenhauer, Klaus (1968): Erziehung und Emanzipation - Polemische Skizzen, München: Juventa.

Moore, David S. (2015): The Developing Genome - An Introduction to Behavioral Epigenetics. Oxford: Oxford University Press.

Rancière, Jacques (2002): Das Unvernehmen: Politik und Philosophie, Frankfurt a.M.: Suhrkamp.

Rancière, Jacques (1991): The Ignorant Schoolmaster, Stanford (CA): Stanford University Press.

Rancière Jacques (2004): The Philosopher and His Poor, Durham: Duke University Press.

Rorty, Richard (1998): Achieving our Country: Leftist Thought in Twenty-Century America, Cambridge MA: Harvard University Press, zit. n. d. deutschen Übersetzung von Hermann Vetter, Frankfurt a.M.: Suhrkamp.

Rorty, Richard (1999): Philosophy and Social Hope. London: Penguin.

Rorty, Richard (1993): »Putnam and the Relativist Menace«, in: Journal of Philosophy 90 (9): S. 443-461.

Rorty, Richard (1998): Das kommunistische Manifest 150 Jahre danach. Frankfurt a.M.: Suhrkamp.

Rousseau, Jean-Jacques (1984): »Diskurs über die Ungleichheit/Discours sur l'inégalité«, in: Heinrich Meier (Hg.): Kritische Ausgabe des integralen Textes: Paderborn: Schöningh [UTB]: S. 79-85.

Rutschky, Katharina (1977): Schwarze Pädagogik, Berlin: Ullstein.

Sartre, Jean- Paul (1967): Kritik der dialektischen Vernunft - Theorie der gesellschaftlichen Praxis. Reinbek: Rowohlt: 273-280,389-388.

Somek, Alexander (2013): »The Individualisation of Liberty: Europe's Move from Emancipation to Empowerment« in: Transnational Legal Theory 4: S. 258-282.

Tugendhat, Ernst (2007): Anthropologie statt Metaphysik, München: Beck.

Weber, Max (1978): Gesammelte Aufsätze zur Religionssoziologie I. Tübingen: Mohr. 
